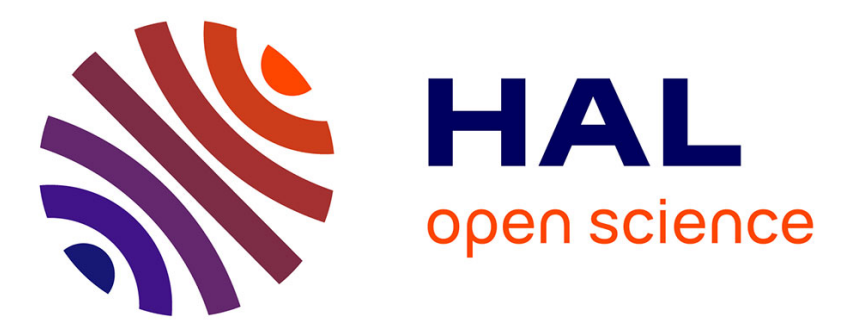

\title{
Probing Exfoliated Graphene Layers and Their Lithiation with Microfocused X-rays
}

Patrik Zielinski, Matthias Kuehne, Daniel Kaercher, Federico Paolucci, Peter Wochner, Sven Fecher, Jakub Drnec, Roberto Felici, Jurgen H. Smet

\section{- To cite this version:}

Patrik Zielinski, Matthias Kuehne, Daniel Kaercher, Federico Paolucci, Peter Wochner, et al.. Probing Exfoliated Graphene Layers and Their Lithiation with Microfocused X-rays. Nano Letters, 2019, 19 (6), pp.3634-3640. 10.1021/acs.nanolett.9b00654 . hal-02976349

\author{
HAL Id: hal-02976349 \\ https://hal.science/hal-02976349
}

Submitted on 23 Oct 2020

HAL is a multi-disciplinary open access archive for the deposit and dissemination of scientific research documents, whether they are published or not. The documents may come from teaching and research institutions in France or abroad, or from public or private research centers.
L'archive ouverte pluridisciplinaire HAL, est destinée au dépôt et à la diffusion de documents scientifiques de niveau recherche, publiés ou non, émanant des établissements d'enseignement et de recherche français ou étrangers, des laboratoires publics ou privés. 


\title{
Probing Exfoliated Graphene Layers and Their Lithiation with Microfocused X-rays
}

\author{
Patrik Zielinski, $^{\dagger}$ Matthias Kühne, ${ }^{\dagger, \S}$ Daniel Kärcher, ${ }^{\dagger}$ Federico Paolucci, ${ }^{\dagger}, \| \odot$ Peter Wochner, ${ }^{\dagger}$
} Sven Fecher, ${ }^{\dagger}$ Jakub Drnec, ${ }^{\ddagger}$ Roberto Felici, ${ }^{\ddagger 0}$ and Jurgen H. Smet ${ }^{*}{ }^{\dagger}$

${ }^{\dagger}$ Max Planck Institute for Solid State Research, 70569 Stuttgart, Germany

${ }^{\ddagger}$ European Synchrotron Radiation Facility (ESRF), 38000 Grenoble, France

\section{Supporting Information}

\begin{abstract}
X-ray diffraction is measured on individual bilayer and multilayer graphene single-crystals and combined with electrochemically induced lithium intercalation. In-plane Bragg peaks are observed by grazing incidence diffraction. Focusing the incident beam down to an area of about $10 \mu \mathrm{m} \times 10 \mu \mathrm{m}$, individual flakes are probed by specular X-ray reflectivity. By deploying a recursive Parratt algorithm to model the experimental data, we gain access to characteristic crystallographic parameters of the samples. Notably, it is possible to directly extract the bi/multilayer graphene $c$-axis lattice parameter. The latter is found to increase upon lithiation, which we control using an on-chip peripheral electrochemical cell layout. These experiments demonstrate the feasibility of in situ X-ray diffraction on individual, micron-sized single crystallites of few- and bilayer two-dimensional materials.
\end{abstract}

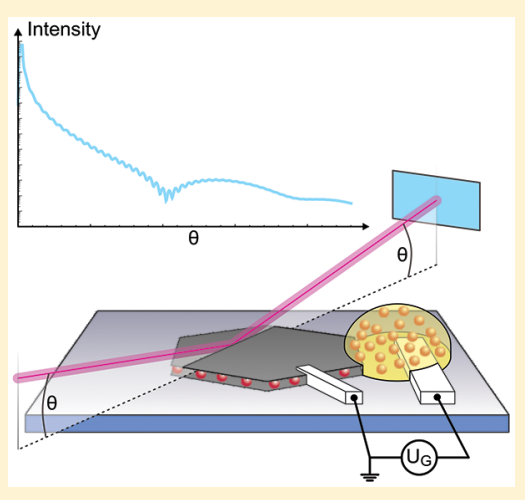

KEYWORDS: Graphene, X-ray diffraction, X-ray reflectivity, intercalation, electrochemistry, lithium

$\mathrm{T}$ wo-dimensional (2D) layered materials attract increasing attention, especially with respect to their manifold incorporation into van der Waals heterostructures. ${ }^{1,2}$ Despite scalable synthesis methods being increasingly put forward, 3,4 mechanical exfoliation from bulk crystals remains the prime route to fabricate such $2 \mathrm{D}$ materials with the highest quality. ${ }^{5}$ The size of devices produced in this manner is however limited and the characteristic lateral dimensions of atomically thin single-crystalline flakes are on the micrometer scale. Because of the tiny interaction volume, samples of this size are extremely challenging to investigate by means of X-ray diffraction (XRD). For the case of graphene, only a few XRD investigations have been reported at the time of this writing. These works have been restricted to thin-film systems with a lateral extent of the atomically thin samples reaching the centimeter scale. Notable examples are the graphene on $\mathrm{SiC}^{6-12}$ as well as the graphene on $\operatorname{Ir}(111)$ system. ${ }^{13}$ In either case, XRD proved to be a sensitive probe to the intercalation of foreign species: hydrogen in graphene on $\mathrm{SiC}^{14}$ oxygen in graphene on $\mathrm{SiC},{ }^{15}$ and cobalt in graphene on $\operatorname{Ir}(111) .{ }^{16}$ More recently, also epitaxial graphene on $\mathrm{Ge}(110)^{17}$ as well as chemical vapor deposited graphene on $\mathrm{SiO}_{2}$-terminated $\mathrm{Si}^{18}$ were investigated by XRD. Here, we go one step further and demonstrate first of all that XRD can indeed be deployed to investigate individual, micron-sized, single-crystalline bilayer or multilayer graphene flakes. In-plane Bragg peaks are measured using grazing incidence X-ray diffraction (GIXD) and out-of-plane signals are captured using specular X-ray reflectivity (XRR; see the schematic in Figure 1a). Combining microfocused XRR with a peripheral electrochemical cell for intercalation, we study the lithiation of individual single-crystals in situ. In a device setup as displayed in Figure $1 \mathrm{~b}, \mathrm{Li}$ ions may intercalate in between carbon sheets and diffuse into the uncovered area of the flake, ${ }^{19}$ here probed by XRR. Using Parratt's recursion formalism, ${ }^{20}$ we extract the associated change in the $c$-axis lattice constant in addition to rich information about each individual sample, such as the thickness, roughness, and density of layers/interfaces at their surface.

This work has been performed on mechanically exfoliated bilayer and multilayer graphene flakes from bulk graphite using adhesive tape. ${ }^{21}$ With the help of a dry transfer technique, ${ }^{22}$ a suitable flake is positioned at the center of a target substrate. We chose $8 \mathrm{~mm} \times 8 \mathrm{~mm} \mathrm{Si}(911)$ substrates terminated by thermally grown $\mathrm{SiO}_{2}$. The extreme spacing between $\mathrm{Si}(911)$ Bragg peaks provides a conveniently large window for investigating graphene X-ray diffraction with minimal spurious substrate contributions. For the same reason, the thickness $d_{\mathrm{SiO}_{2}}$ of the amorphous $\mathrm{SiO}_{2}$ was kept to a minimum; here, it was on the order of $10 \mathrm{~nm}$. This is nonetheless still thick enough to serve as a reliable electric insulator and also exceeds the thickness of the graphene flake to be investigated. The selected flake is isolated from other flakes initially present on the substrate by PMMA peeling, so it remains the only graphene on the entire substrate. ${ }^{23}$ Electron beam lithography

Received: February 14, 2019

Revised: April 23, 2019

Published: May 16, 2019 


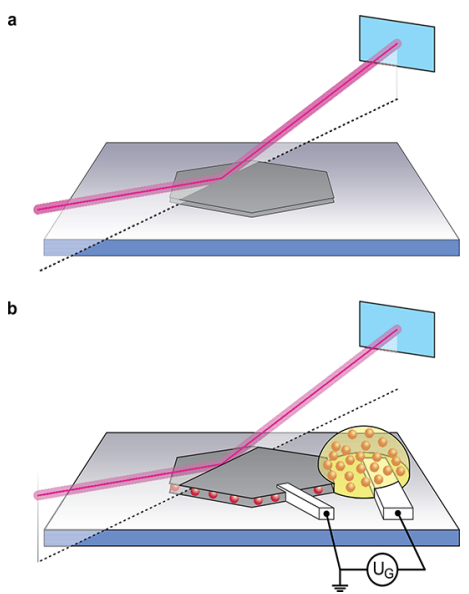

Figure 1. (a) Schematic device layout of our X-ray diffraction experiments on exfoliated graphene layers on $\mathrm{Si}$ substrates. (b) Schematic experimental configuration for in situ X-ray diffraction investigations on the electrochemical lithiation of exfoliated graphene flakes. For illustration purposes, here we show the case of single crystal bilayer graphene. A solidified electrolyte (yellow) serves as a source of Li-ions (red spheres) that may intercalate in between the two graphene sheets (dark gray) when applying a gate voltage $U_{\mathrm{G}}$ to a counter electrode with respect to the bilayer. During this process, microfocused X-rays (violet) may be diffracted from the sample to probe structural changes. The intensity of diffracted X-rays is measured by a detector (cyan).

and lift-off techniques are used to create a set of markers out of $5 \mathrm{~nm} \mathrm{Ti}$ and $30 \mathrm{~nm} \mathrm{Au}$ near the center of the substrate. They facilitate both fine-positioning of the X-ray beam using Au fluorescence as well as the additional processing steps required to complete the device as explained below.

Grazing Incidence X-ray Diffraction. GIXD was measured at the MPI beamline of the Angströmquelle
Karlsruhe (ANKA). Two sets of experiments were performed with incident X-ray beam energies of $10 \mathrm{keV}(\lambda=1.240 \AA)$ and $12 \mathrm{keV}(\lambda=1.033 \AA)$, respectively. The incident X-ray beam profile was focused to the size of approximately $300 \mu \mathrm{m} \times 150$ $\mu \mathrm{m}$ (width $\times$ height). The mounted sample was enclosed with a Kapton dome and flushed with $\mathrm{He}$ gas. Figure 2a displays a schematic of the GIXD experiment. The detector is set at an angle of $2 \theta$ with respect to the incident synchrotron light beam, such that the difference between $k_{i}$ and $\boldsymbol{k}_{f}$ matches an inplane Bragg peak of the graphene flake. $k_{i}$ and $\boldsymbol{k}_{f}$ are the wave vectors of the incident and diffracted light, respectively. $\theta$ was determined using the quadratic form of the Bragg equation for a hexagonal crystal

$$
\sin ^{2} \theta=\frac{\lambda^{2}}{4}\left(\frac{4}{3} \frac{h^{2}+h k+k^{2}}{a^{2}}+\frac{l^{2}}{c^{2}}\right)
$$

Here, $\lambda$ is the X-ray wavelength, $a(c)$ is the in-plane (out-ofplane) lattice constant and $h k l$ are the Miller indices. With $a=$ $2.46 \AA$ and $\lambda=1.240 \AA$, the most prominent (100) Bragg peak should be observed at $\theta_{100}=16.92^{\circ}$. As shown in Figure 2a, with the detector set at a fixed angle of $2 \theta_{100}$ with respect to the incident beam, the sample is rotated around the $c$-axis of the graphene flake. For a $60 \mathrm{~nm}$ thick graphene multilayer, Bragg reflection from the (100) planes is readily measured (Figure 2b). The appearance of only one family of peaks demonstrates the single-crystalline nature of the flake. Figure $2 \mathrm{~d}$ illustrates GIXD data measured on a bilayer graphene flake. Despite the less favorable signal-to-noise ratio, the $60^{\circ}$ in-plane diffraction symmetry of the equivalent $(100) /(1 \overline{1} 0)$ reflections is nonetheless observed and covers a full $180^{\circ}$-range in rotation about the bilayer $c$-axis. Since there is only one such set of reflections, this graphene bilayer crystal also consists of a single domain. Comparing the measured data with the sample alignment on the diffractometer, the orientation of the bilayer graphene lattice with respect to the substrate, modulo an angle
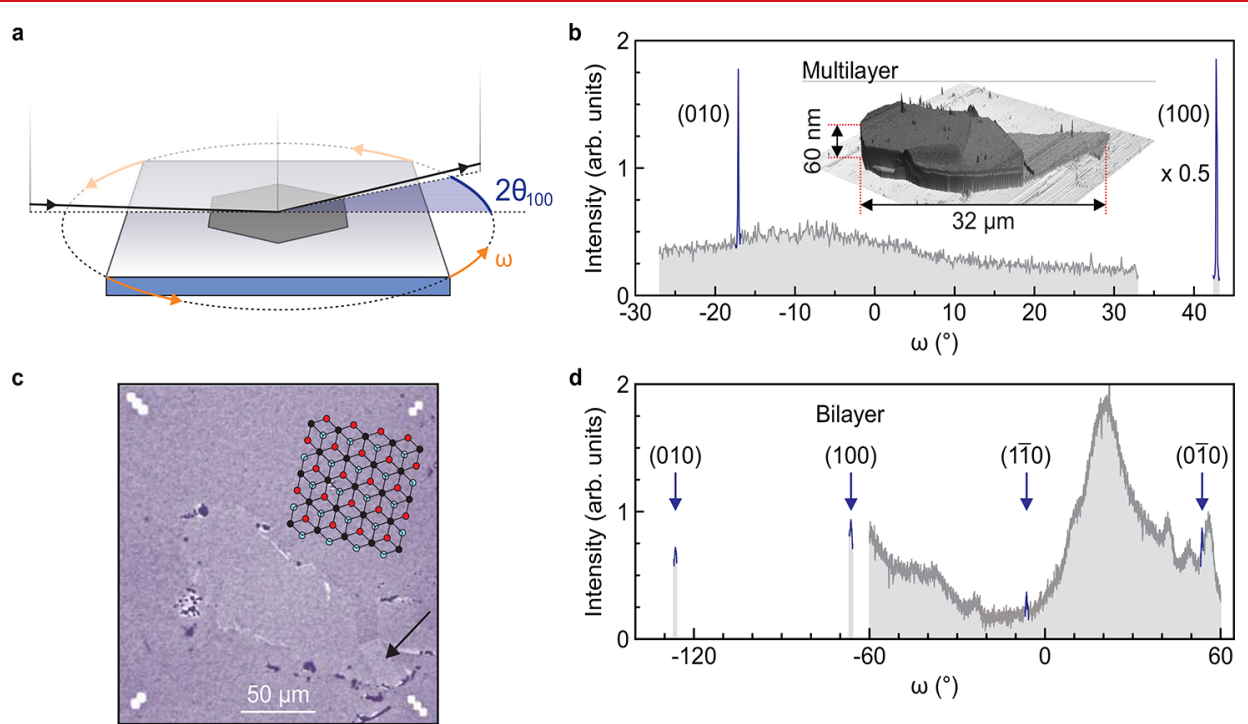

Figure 2. GIXD. (a) Schematic of the measurement with the detector set at a fixed in-plane angle $2 \theta_{100}$ (blue) with respect to the grazing incident $\mathrm{X}$-ray beam. The sample is slowly rotated about $\omega$, indicated by the orange arrows. Data measured on (b) a graphene multilayer and (d) a graphene bilayer on $\mathrm{Si}(911)$. Highlighted in blue are the measured Bragg peaks. The strongly $\omega$-dependent background in (d) is largely due to a significant excentricity of the flake on its substrate. Inset in (b) is a three-dimensional rendering of an atomic force micrograph of the multilayer. (c) Optical micrograph of the bilayer graphene flake on which the data in $(d)$ was measured. Four gold markers can be seen near the corners of the micrograph. The inset shows the orientation of the $\mathrm{AB}$-stacked bilayer lattice modulo $60^{\circ}$ with respect to the substrate deduced from (d) and considering our diffractometer setup. The black arrow marks a folded part of the flake from which no diffraction signals are observed. 
a

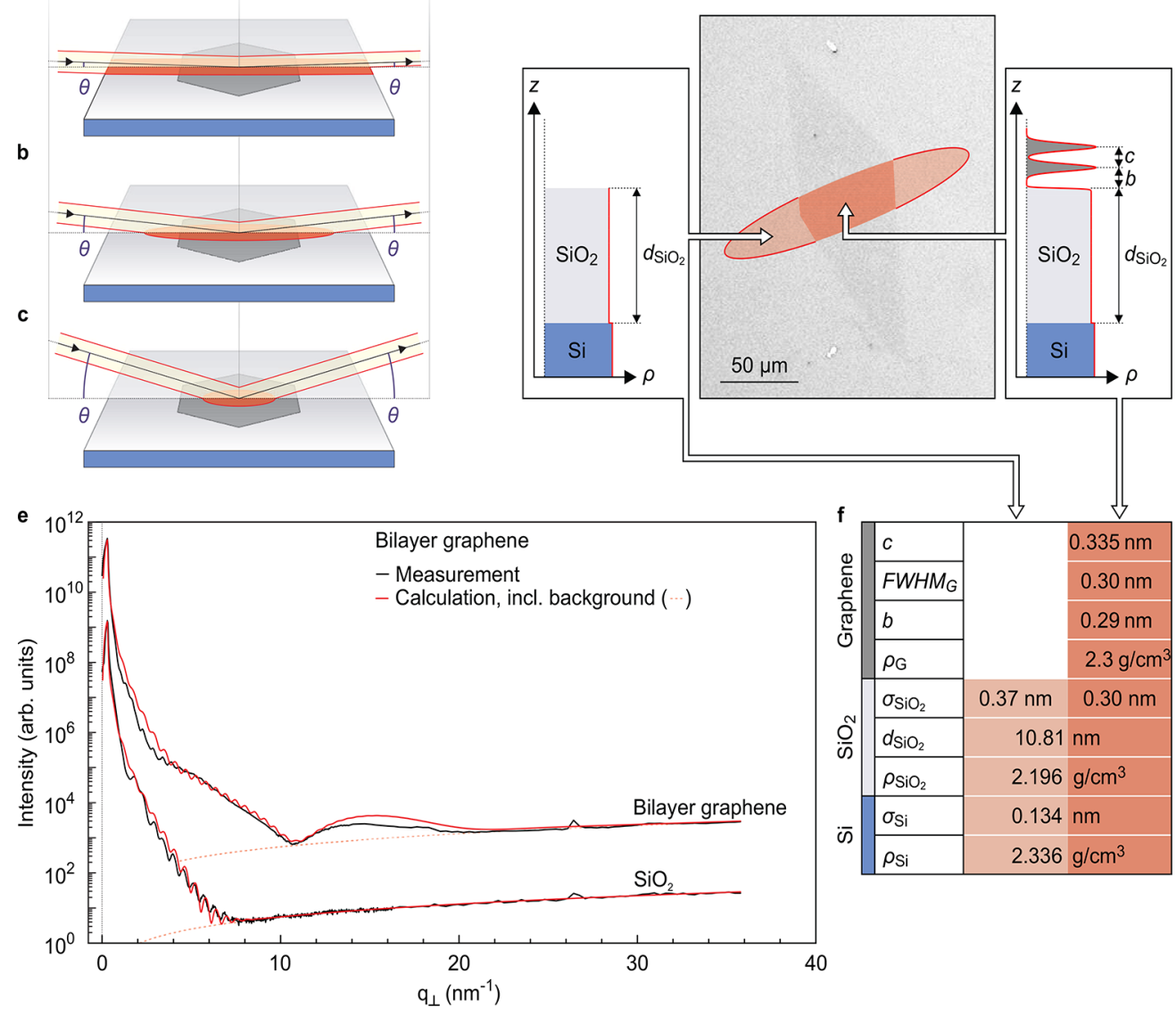

Figure 3. Specular XRR. $(a-c)$ Schematic of the measurement with increasing angle $\theta$ from (a) to (c) leading to a varying footprint (red ellipse) of the X-ray beam (black arrow; a finite vertical extent illustrated by red lines enclosing a yellow shaded area) on the sample: a graphene flake (dark gray hexagon) on $\mathrm{SiO}_{2}$ (light gray). (d) Optical micrograph of a probed bilayer graphene flake on $\mathrm{SiO}_{2}$-terminated $\mathrm{Si}(911)$ (central panel). The red ellipse illustrates the footprint of the X-ray beam at a given value of $\theta$. In the side panels, we schematically show $z$-dependent density profiles for the probed parts of the sample that are either covered by the graphene flake or uncovered. (e) XRR data measured on the bare substrate (lower black curve) and on bilayer graphene (upper black curve). Curves calculated using Parratt's recursion formalism with parameters stated in (f) are superposed (red), which further includes a second order polynomial background (dashed lines). The two data sets in (e) are offset for clarity. Difference curves are contained in the Supporting Information.

of $60^{\circ}$, can be deduced. A properly rotated schematic of the bilayer crystal structure is superposed onto the optical micrograph of the sample in Figure $2 \mathrm{c}$. Note that the small, folded part of the bilayer marked by the black arrow in the micrograph does not give rise to a second, rotated set of (100) Bragg peaks in Figure 2d. This absence is attributed to its small size and because it is possibly probed by the less intense flank of the X-ray beam. It highlights the need of high beam intensity as well as optimized detector conditions when investigating such micron-sized, atomically thin crystallites.

Specular X-ray Reflectivity. Specular XRR was measured at the ID03 surface diffraction beamline of the European Synchrotron Radiation Facility (ESRF). Two sets of experiments were performed with incident X-ray beam energies of 12 $\operatorname{keV}(\lambda=1.033 \AA)$ and $18.5 \mathrm{keV}(\lambda=0.670 \AA)$, respectively. Once mounted on the six-circle diffractometer, the sample was enclosed in a Be dome evacuated to a pressure of $p<10^{-5}$ mbar. In Figure $3 a-c$, a schematic of the XRR experiment is shown. The intensity of the reflected beam is recorded as a function of $\theta$, where $\theta$ is the angle of both incident and reflected beam with respect to the sample surface. The footprint of the X-ray beam on the sample (illustrated by the red ellipse) depends on the value of $\theta$. At the lowest values of $\theta$ this footprint exceeds the width of both the graphene flake and the substrate (Figure 3a), at intermediate values it exceeds only the width of the graphene flake (Figure $3 b$ ), and at higher values it is entirely contained within the graphene flake (Figure $3 c)$.

The central panel of Figure $3 \mathrm{~d}$ is the optical micrograph of the bilayer graphene flake studied by XRR. Here, $\lambda$ equals $0.670 \AA$ and the incident $\mathrm{X}$-ray beam was focused to the size (width $\times$ height) of $11.5 \mu \mathrm{m} \times 13 \mu \mathrm{m}$. In Figure $3 \mathrm{e}$, experimental data for two XRR scans are depicted (black): one centered on the graphene bilayer shown in panel $\mathrm{d}$ and one outside the flake, that is, centered on the bare $\mathrm{SiO}_{2}$-terminated $\mathrm{Si}(911)$ substrate. The measured intensity is plotted as a function of momentum transfer perpendicular to the surface $q_{\perp}$ $=4 \pi \lambda^{-1} \sin \theta$. The two data sets are offset for clarity and show both characteristic similarities and differences. We first consider the lower data set obtained from the XRR scan on the bare substrate. As illustrated in Figure 3a, the intensity increase with increasing values of $q_{\perp}$ for $q_{\perp}<q_{\mathrm{c}}$ is due to the finite sample dimensions with a growing portion of the X-ray beam actually hitting the sample surface and being reflected toward the detector. The value of $q_{c}$ is determined by the critical angle of total reflection $\theta_{c}$ of $\mathrm{SiO}_{2}$. For $q_{\perp}>q_{\mathrm{c}}$ one 
observes a strong, asymptotic decrease in intensity, following an overall $\left(2 q_{\perp} / q_{\mathrm{c}}\right)^{-4}$ behavior commonly referred to as Fresnel reflectivity. ${ }^{24}$ At larger values of $q_{\perp}$, the intensity is found to rise again with increasing $q_{\perp}$ : a background stemming largely from the tail of a Bragg peak appearing beyond the measurement range. At intermediate values of $q_{\perp}$, interference fringes with a spacing between neighboring extrema on the order of $\Delta q_{\perp}=0.6 \mathrm{~nm}^{-1}$ are observed. These so-called Kiessig fringes stem from the interference of $\mathrm{X}$-rays reflected from the top $\mathrm{SiO}_{2}$ surface of the substrate as well as the $\mathrm{Si} / \mathrm{SiO}_{2}$ interface buried about $10 \mathrm{~nm}$ below. The damping of the amplitude of these fringes toward higher values of $q_{\perp}$ points to a finite roughness of the $\mathrm{Si} / \mathrm{SiO}_{2}$ interface. The upper curve in Figure 3e, now measured with the beam focused on bilayer graphene, retains most of the findings pointed out so far. At first glance, however, one also notes an additional contribution to the reflectivity, pronounced especially at $2 \mathrm{~nm}^{-1}<q_{\perp}<20$ $\mathrm{nm}^{-1}$, that stems from the bilayer graphene flake.

In order to fully appreciate the amount of information that can be extracted from the experimental data, we model our sample's XRR using Parratt's recursion formalism. ${ }^{20}$ This approach, discussed at length in the Supporting Information, describes the specular reflectivity of a multilayer structure consisting of slices of varying thickness and density on top of an infinitely thick substrate. It also allows accounting for the roughness of each interface. Here, XRR contributions from the irradiated part of the graphene flake and of the bare $\mathrm{SiO}_{2}$ substrate are considered. Their corresponding density profiles are taken into account as schematically shown in the side panels of Figure 3d. Parameters are the densities $\rho_{\mathrm{Si}}$ of $\mathrm{Si}, \rho_{\mathrm{SiO}_{2}}$ of $\mathrm{SiO}_{2}, \rho_{\mathrm{G}}$ of graphitic carbon, the roughnesses $\sigma_{\mathrm{Si}}$ of $\mathrm{Si}, \sigma_{\mathrm{SiO}_{2}}$ of $\mathrm{SiO}_{2}, \sigma_{\mathrm{G}}$ of graphene, the thickness $d_{\mathrm{SiO}_{2}}$ of $\mathrm{SiO}_{2}$, the separation $b$ between the lowest graphene layer and the $\mathrm{SiO}_{2}$ surface, as well as the graphene flake's $c$-axis lattice spacing $c$. The density distribution of each graphene sheet is approximated as a Gaussian characterized by a full width at halfmaximum $\left(\mathrm{fwhm}_{\mathrm{G}}\right)$ of $0.3 \mathrm{~nm}$ (accounting for finite corrugation of the carbon sheets). Roughness values effectively describe a root-mean-square density variation at the top of the respective layer. The $\theta$-dependent footprint of the X-ray beam on the sample is also included in the model (illustrated in Figure $3 \mathrm{a}-\mathrm{c})$. The high $-\theta$ background is accounted for by a second order polynomial (dashed lines). By calculating the XRR response measured on the bare substrate first (lower red curve in Figure 3e), the parameters for $\mathrm{Si}$ and $\mathrm{SiO}_{2}$ are extracted. These values are taken into account when computing the XRR response of the bilayer graphene flake (upper red curve in Figure 3e). Intensities are summed in a coherent fashion. Additional information about the calculations can be found in the Supporting Information.

From the calculated XRR of the bare substrate, we obtain $d_{\mathrm{SiO}_{2}}=10.81 \mathrm{~nm}$, given by the spacing between extrema of the Kiessig fringes. Their amplitude yields $\rho_{\mathrm{SiO}_{2}}=2.196 \mathrm{~g} / \mathrm{cm}^{3}$ that is in close agreement with the literature value of $2.202 \mathrm{~g} /$ $\mathrm{cm}^{3}{ }^{25}$ The surface roughness of this layer $\sigma_{\mathrm{SiO}_{2}}=0.37 \mathrm{~nm}$ (comparable to the findings of ref 26) is largely responsible for the $q_{\perp}$-dependent drop in signal intensity beyond $q_{c}$. In addition, the slope of the intensity drop depends on the density of the underlying Si layer, which we extract as $\rho_{\mathrm{Si}}=$ $2.336 \mathrm{~g} / \mathrm{cm}^{3}$. This is in good agreement with the literature value of $2.329 \mathrm{~g} / \mathrm{cm}^{3}{ }^{25}$ A finite surface roughness of $\mathrm{Si}, \sigma_{\mathrm{Si}}=$
$0.134 \mathrm{~nm}$, reproduces well the $q_{\perp}$-dependent damping of the Kiessig fringes. Overall, the observed behavior is characteristic of $\mathrm{SiO}_{2}$-terminated $\mathrm{Si}$ substrates (see, for example, refs 27 and 28). These parameters are kept to analyze the XRR response of bilayer graphene (upper data set in Figure 3e). Good agreement with the data is obtained by assuming the density $\rho_{\mathrm{G}}=2.3 \mathrm{~g} / \mathrm{cm}^{3}$ of the graphene flake, which compares well with the literature value for bulk graphite of $2.25 \mathrm{~g} / \mathrm{cm}^{3}{ }^{25} \mathrm{We}$ also determine the spacing between the two carbon sheets $c=$ $0.335 \pm 0.003 \mathrm{~nm}$, a typical value for $\mathrm{AB}$-stacked bilayer graphene. ${ }^{29}$ We estimate the error comparing our data with a set of XRR curves calculated for values of $c$ closely spaced around the optimal value. For the preceding analysis, the geometry of the probed sample is taken into account (Figure $3 \mathrm{~d}$ ) and an approximately elliptic X-ray beam profile with a vertical semiaxis $h=6.5 \mu \mathrm{m}$ is assumed. We systematically achieve an overall better match between our calculations and the experiment by considering conformation of graphene to the $\mathrm{SiO}_{2}$ surface roughness (see Supporting Information). ${ }^{26,30}$ Here, a slightly lower value of $\sigma_{\mathrm{SiO}_{2}}=0.3 \mathrm{~nm}$ has been allowed for to obtain the plotted result.

Lithiation. To intercalate a graphene flake with lithium, an electrochemical cell is created at the flake's perimeter. To this end, $60 \mathrm{~nm}$ thick Ti leads were fabricated on top of the sample using electron beam lithography combined with lift-off techniques. The Ti electrodes serve either as contacts to the graphene flake or as the counter electrode of the cell. A Li-ion conducting polymer electrolyte ${ }^{31}$ drop is placed on top of the substrate inside an Ar-filled glovebox such that it covers the bilayer graphene flake only partially. ${ }^{19,32}$ To facilitate the positioning, here we exploit surface wettability engineering. It stops the advancing (during drop casting) rim of the electrolyte drop at the interface between $\mathrm{SiO}_{2}$ and a lithographically patterned area that is rendered more hydrophobic by silanization. Care was taken not to silanize parts of the sample surface that are probed by X-ray diffraction. Eventually, the electrolyte, $0.35 \mathrm{M}$ lithium bis(trifluoromethane) sulfonimide in polyethylene glycol methyl ether methacrylate/bisphenol A ethoxylate dimethacrylate $\mathrm{w} / \mathrm{w}$ 3:7 with an added 2-4 wt \% of 2-hydroxy-2-methylpropiophenone (a common photoinitiator), is solidified by ultraviolet-curing. A schematic of the device layout is displayed in Figure $1 \mathrm{~b}$.

Bi- and multilayer graphene flakes are lithiated through potentiostatic polarization by applying a gate voltage $U_{\mathrm{G}}$ on the order of several volt across the $\mathrm{Li}$-ion conducting electrolyte between the counter electrode and the graphene flake. This forces Li-ions to intercalate the graphene sheets. Experiments conducted in a similar configuration in ref 19 have demonstrated that Li-ions only enter in between the graphene sheets and diffusion on top of the bilayer flake or in between the bilayer and the $\mathrm{SiO}_{2}$ is not relevant. Once intercalated, $\mathrm{Li}$ ions were shown to perform rapid lateral diffusion in order to minimize concentration gradients within the flake. In this way, also the uncovered (from the electrolyte) part of the flake gets filled by Li-ions. Here, in situ XRR is measured by focusing the incident X-ray beam onto this uncovered part of the flake as schematically shown in Figure $1 \mathrm{~b}$. By having the beam pass alongside (and not through) the electrolyte, the amount of beam damage potentially inflicted is minimized. To reveal crystallographic changes induced by the insertion of Li-ions in between graphene sheets, XRR data is recorded both before and during lithiation. 
Figure 4 shows XRR data recorded on a graphene bilayer before and during lithiation at $U_{G}=8 \mathrm{~V}$ (black). Here, $\lambda=$

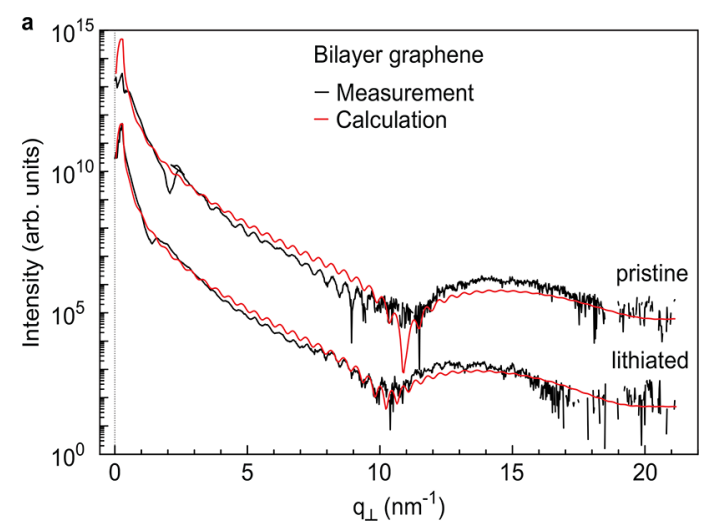

b

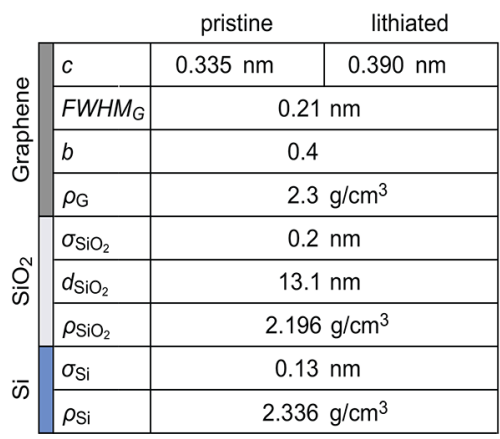

Figure 4. (a) Specular XRR before (upper curves) and during (lower curves, $U_{\mathrm{G}}=8 \mathrm{~V}$ ) lithiation of a bilayer graphene flake (normalized by the intensity of the incident X-ray beam). Experimental data (black) and curves calculated using Parratt's recursion formalism (red) with the parameters given in (b). The two data sets in (a) are offset for clarity. Difference curves are contained in the Supporting Information.

$1.033 \AA$ and the incident $\mathrm{X}$-ray beam was focused to an area of $14 \mu \mathrm{m} \times 15 \mu \mathrm{m}$. From the calculated XRR (red solid lines), a distance between graphene sheets $c$ of $0.335 \pm 0.005 \mathrm{~nm}$ for the pristine flake and $c^{\prime}=0.390 \pm 0.005 \mathrm{~nm}$ for the lithiated one is extracted. The latter value exceeds the typical range of interlayer spacings $0.335 \mathrm{~nm} \leq c \leq 0.37 \mathrm{~nm}$ achieved in bulk $\mathrm{Li}_{x} \mathrm{C}_{6}$ with $0 \leq x \leq 1 .^{33,34}$ Quantitatively, $c^{\prime}$ might be overestimated due to the difficulty in precisely reproducing the apparent shift between both data sets in Figure 4a at $q_{\perp}>8$ $\mathrm{nm}^{-1}$. Yet, qualitatively the stated value of $c^{\prime}$ points to a high $\mathrm{Li}$ content, possibly in moderate excess of the $\mathrm{LiC}_{6}$ stoichiometry. Alternative intercalant ordering as recently observed by in situ transmission electron microscopy could be an explanation. ${ }^{32}$ For the calculation, we assume the density values for $\mathrm{Si}$ and $\mathrm{SiO}_{2}$ as in Figure 3f. Also for graphite the value of $\rho_{\mathrm{G}}=2.3 \mathrm{~g} /$ $\mathrm{cm}^{3}$ is kept, because intercalated $\mathrm{Li}$ causes only a minor deviation in the total density. In fact, for $\mathrm{LiC}_{6}$ a very similar density of $\rho_{\mathrm{LiC} 6}=2.24 \mathrm{~g} / \mathrm{cm}^{3}$ is reported. ${ }^{35}$ Each graphene layer's $z$-dependent density distribution is taken care of by assuming a Gaussian with $\mathrm{FWHM}_{\mathrm{G}}=0.21 \mathrm{~nm}$. For this sample, we also find $d_{\mathrm{SiO}_{2}}=13.1 \mathrm{~nm}$ to yield a better agreement between the calculated curve and the observed Kiessig fringes. A local minimum in their amplitude near $q_{\perp}=$ $6 \mathrm{~nm}^{-1}$ in the top data set in Figure $4 \mathrm{a}$ is not clearly reproduced in the lower one. It might be due to beating with XRR from beam-induced contamination of comparable thickness during acquisition (see Supporting Information; a similar feature is present in the top data set of Figure $3 \mathrm{e}$ around $\left.q_{\perp}=5 \mathrm{~nm}^{-1}\right)$. Drifts in the beam alignment are responsible for unreproducible artifacts in the acquired data, notably at $q_{\perp}<3 \mathrm{~nm}^{-1}$.

Figure 5 shows XRR data acquired on a 10 layer graphene flake before and during lithiation at $U_{\mathrm{G}}=5 \mathrm{~V}$ (black). Here, $\lambda$

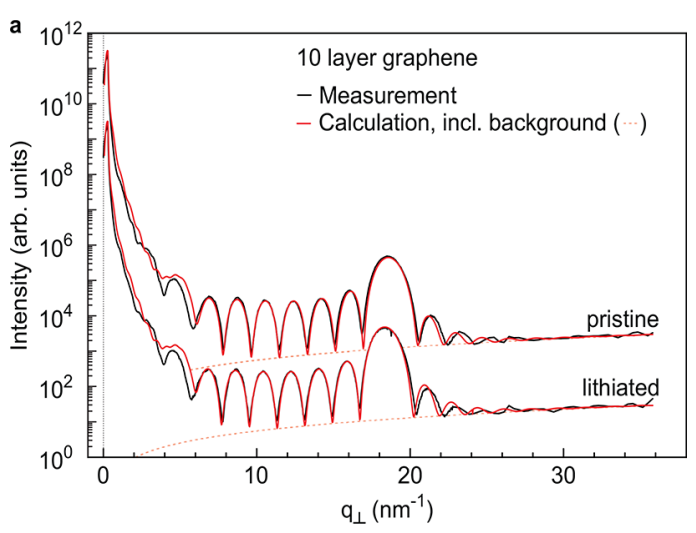

b

\begin{tabular}{|c|c|c|c|}
\hline \multirow{5}{*}{ 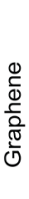 } & & pristine & lithiated \\
\hline & $c$ & $0.335 \mathrm{~nm}$ & $0.34 \mathrm{~nm}$ \\
\hline & $F W H M_{G}$ & \multicolumn{2}{|c|}{$0.20 \mathrm{~nm}$} \\
\hline & $b$ & \multicolumn{2}{|c|}{$0.27 \mathrm{~nm}$} \\
\hline & $\rho_{\mathrm{G}}$ & \multicolumn{2}{|c|}{$2.3 \mathrm{~g} / \mathrm{cm}^{3}$} \\
\hline & $\sigma_{\mathrm{SiO}_{2}}$ & \multicolumn{2}{|c|}{$0.30 \mathrm{~nm}$} \\
\hline 음 & $d_{\mathrm{SiO}_{2}}$ & \multicolumn{2}{|c|}{$10.81 \mathrm{~nm}$} \\
\hline & $\rho_{\mathrm{SiO}_{2}}$ & \multicolumn{2}{|c|}{$2.196 \mathrm{~g} / \mathrm{cm}^{3}$} \\
\hline & $\sigma_{\mathrm{Si}}$ & \multicolumn{2}{|c|}{$0.35 \mathrm{~nm}$} \\
\hline & $\rho_{\mathrm{Si}}$ & \multicolumn{2}{|c|}{$2.336 \mathrm{~g} / \mathrm{cm}^{3}$} \\
\hline
\end{tabular}

Figure 5. (a) Specular XRR before (upper curves) and during (lower curves, $U_{\mathrm{G}}=5 \mathrm{~V}$ ) lithiation of a 10 layer graphene flake (normalized by the intensity of the incident X-ray beam). Experimental data (black) and curves calculated using Parratt's recursion formalism (red) with the parameters given in (b). Solid red lines include a second order polynomial background (dashed lines). The two data sets in (a) are offset for clarity. Difference curves are contained in the Supporting Information.

$=0.670 \AA$ and the incident X-ray beam was focused to an area of $11.5 \mu \mathrm{m} \times 11 \mu \mathrm{m}$. In contrast to the bilayer case, we observe most prominently a Bragg peak near $q_{001}=19 \mathrm{~nm}^{-1}$. From the calculated XRR curves (red), we extract the average spacing between carbon sheets $c=0.335 \pm 0.001 \mathrm{~nm}$ for the pristine flake and $c^{\prime}=0.340 \pm 0.001 \mathrm{~nm}$ for the lithiated one. Note that here we do not resolve any heterogeneity in $c$ that could potentially be induced by staging, a periodic ordering of the $\mathrm{Li}$ intercalate in $c$-axis direction that leaves some of the interlayer spaces unoccupied. However, the small difference in $c$ due to lithiation suggests dilute intercalation only, for which no stage ordering is to be expected. ${ }^{36}$ Although the dependence of the interlayer spacing $c$ as a function of $x$ in $\mathrm{Li}_{x} \mathrm{C}_{6}$ (with $0 \leq x \leq 1$ ) violates Vegard's law, ${ }^{33,34,37}$ an approximate stoichiometry of $\mathrm{Li}_{0.05} \mathrm{C}_{6}$ can be estimated by comparing the observed increase $\Delta c=0.005 \mathrm{~nm}$ to neutron powder diffraction data. ${ }^{37}$ Note that $\mathrm{XRR}$ is insensitive to changes in interlayer stacking. For the calculation, we again assume $\rho_{\mathrm{Si}}, \rho_{\mathrm{SiO}_{2}}$, and $\rho_{\mathrm{G}}$ as before (Figure 3f). For this sample, $d_{\mathrm{SiO}_{2}}$ as well as the $\mathrm{Si} / \mathrm{SiO}_{2}$ interface roughness $\sigma_{\mathrm{Si}}$ are difficult to determine as the $\mathrm{SiO}_{2}$-related 
Kiessig fringes are hard to discern. The $z$-dependent density distributions of graphene sheets are modeled as Gaussians with a $\mathrm{FWHM}_{\mathrm{G}}$ equal to $0.2 \mathrm{~nm}$. Note that variations in $\mathrm{FWHM}_{\mathrm{G}}$ and $b$ among samples are most probably due to variations in $\mathrm{SiO}_{2}$ surface conditions during device fabrication; see also ref 38.

Discussion and Conclusion. We have demonstrated the feasibility of measuring XRD on individual micron-sized singlecrystals of bi- and multilayered graphene flakes supported on a substrate. The structural properties of such ultrathin crystalline layers were directly investigated and we believe this can likely even be accomplished down to the limit of a single atomic layer. Characteristic in-plane Bragg peaks can be observed by GIXD, whereas the $c$-axis lattice spacing can be probed by XRR. These approaches can in principle be extended to micron-sized crystalline layers of any other material, including those routinely produced nowadays by mechanical exfoliation of layered compounds. ${ }^{5}$ Also van der Waals heterostructures, stacked sets of different atomic layers, ${ }^{1,2}$ may be considered to extract precise structural information including their mutual orientation, which remains often elusive otherwise. Identifying a substrate with minimal spurious XRD contributions in the range of the desired signal as well as making sure that only the target flake is probed are challenging, however we have shown that these issues can be overcome. Advanced focusing optics connected to a highly brilliant $\mathrm{X}$-ray source as well as isolating $^{23}$ a transferred flake to ensure that it is the only one on the entire substrate are conducive factors that have been exploited here. The characteristic time scales of a particular XRD experiment as well as the potentially invasive effects of the irradiation remain possible limitations. By implementing an optimized on-chip electrochemical cell layout, we have succeeded in performing in situ XRD during the lithiation of individual graphene single-crystals without exposing the electrolyte to the X-ray beam. By analyzing our XRR data with a model based on Parratt's recursion formalism, ${ }^{20}$ an enhancement of the interlayer separation $c$ due to the intercalation of Li-ions between graphene sheets has been successfully detected. Even if XRD is an established tool for research on intercalation compounds, ${ }^{39}$ this work constitutes an advance since the initial in situ efforts of Dahn et al. ${ }^{34,40}$ toward studying the intercalation of $\mathrm{Li}$ in graphitic carbon at the limit of pristine, atomically thin singlecrystals. We note that the varying amount of intercalated $\mathrm{Li}$ inferable from data given in Figures 4 and 5 is probably due to deficiencies in our electrochemical setup, especially the lack of a lithium metal counter/reference electrode with the help of which $\mathrm{Li}$ intercalation would be more determinable. For technical reasons, a lithium electrode could however not be implemented. Also sample-specific imperfections of the graphene flakes such as wrinkles that hinder $\mathrm{Li}$ diffusion or edges/defects that provide escape routes for intercalated $\mathrm{Li}$ may play an important role preventing the insertion of additional $\mathrm{Li}^{19,41}$ We note that the characteristic acquisition time for an XRR scan, as given in Figures 3e, 4a, and 5a, was on the order of $30 \mathrm{~min}$, a time frame during which the sample's state of lithiation may vary. Although there is certainly room for more refined experiments at improved conditions, even with the present state of affairs one may target XRD studies during the intercalation of other 2D layered materials ${ }^{42}$ or even heterostructures. ${ }^{43,44}$ Beyond electrochemistry, it also becomes conceivable to probe structural changes in such materials in situ, initiated by entirely different forces such as, for example, the application of external strain.

\section{ASSOCIATED CONTENT}

\section{Supporting Information}

The Supporting Information is available free of charge on the ACS Publications website at DOI: 10.1021/acs.nanolett.9b00654.

Modeling specular XRR; beam-induced contamination (PDF)

\section{AUTHOR INFORMATION}

\section{Corresponding Author}

*E-mail: j.smet@fkf.mpg.de.

ORCID

Federico Paolucci: 0000-0001-8354-4975

Roberto Felici: 0000-0001-9897-5866

Jurgen H. Smet: 0000-0002-4719-8873

\section{Present Addresses}

$\S$ (M.K.) Department of Chemical Engineering, Massachusetts Institute of Technology, Cambridge, Massachusetts 02139, U.S.A.

"(F.P.) INFN Sezione di Pisa, 56127 Pisa, Italy.

\section{Notes}

The authors declare no competing financial interest.

\section{ACKNOWLEDGMENTS}

We acknowledge financial support from the Graphene Flagship. We thank B. Keimer as well as $\mathrm{K}$. von Klitzing for support and discussions. We are further grateful to $\mathrm{H}$. Reichert for discussions, U. Zschieschang for silanization, as well as $\mathrm{O}$. Seeck and co-workers at PETRA III (DESY) for preliminary experiments and optical microscopy equipment. We also thank E. Benckiser for useful comments on the manuscript. We acknowledge the Synchrotron Light Source ANKA for provision of the beam. XRR experiments were performed on beamline ID03 at the European Synchrotron Radiation Facility (ESRF), Grenoble, France.

\section{REFERENCES}

(1) Novoselov, K. S.; Mishchenko, A.; Carvalho, A.; Castro Neto, A. H. 2D materials and van der Waals heterostructures. Science 2016, 353, aac9439.

(2) Liu, Y.; Weiss, N. O.; Duan, X.; Cheng, H.-C.; Huang, Y.; Duan, X. Van der Waals heterostructures and devices. Nat. Rev. Mater. 2016, $1,16042$.

(3) Lin, Z. 2D materials advances: from large scale synthesis and controlled heterostructures to improved characterization techniques, defects and applications. 2D Mater. 2016, 3, 042001.

(4) Kang, K.; Lee, K.-H.; Han, Y.; Gao, H.; Xie, S.; Muller, D. A.; Park, J. Layer-by-layer assembly of two-dimensional materials into wafer-scale heterostructures. Nature 2017, 550, 229-233.

(5) Novoselov, K. S.; Jiang, D.; Schedin, F.; Booth, T. J.; Khotkevich, V. V.; Morozov, S. V.; Geim, A. K. Two-dimensional atomic crystals. Proc. Natl. Acad. Sci. U. S. A. 2005, 102, 10451-10453.

(6) Varchon, F.; Feng, R.; Hass, J.; Li, X.; Nguyen, B. N.; Naud, C.; Mallet, P.; Veuillen, J.-Y.; Berger, C.; Conrad, E. H.; Magaud, L. Electronic Structure of Epitaxial Graphene Layers on SiC: Effect of the Substrate. Phys. Rev. Lett. 2007, 99, 126805.

(7) Hass, J.; Feng, R.; Millán-Otoya, J. E.; Li, X.; Sprinkle, M.; First, P. N.; de Heer, W. A.; Conrad, E. H.; Berger, C. Structural properties of the multilayer graphene $/ 4 \mathrm{H}-\mathrm{SiC}(000 \overline{1})$ system as determined by 
surface x-ray diffraction. Phys. Rev. B: Condens. Matter Mater. Phys. 2007, 75, 214109.

(8) Hass, J.; Millán-Otoya, J. E.; First, P. N.; Conrad, E. H. Interface structure of epitaxial graphene grown on $4 \mathrm{H}-\mathrm{SiC}(0001)$. Phys. Rev. B: Condens. Matter Mater. Phys. 2008, 78, 205424.

(9) Emery, J. D.; Wang, Q. H.; Zarrouati, M.; Fenter, P.; Hersam, M. C.; Bedzyk, M. J. Structural analysis of PTCDA monolayers on epitaxial graphene with ultra-high vacuum scanning tunneling microscopy and high-resolution X-ray reflectivity. Surf. Sci. 2011, 605, 1685-1693.

(10) Zhou, H.; Ganesh, P.; Presser, V.; Wander, M. C. F.; Fenter, P.; Kent, P. R. C.; Jiang, D.; Chialvo, A. A.; McDonough, J.; Shuford, K. L.; Gogotsi, Y. Understanding controls on interfacial wetting at epitaxial graphene: Experiment and theory. Phys. Rev. B: Condens. Matter Mater. Phys. 2012, 85, 035406.

(11) Emery, J. D.; Detlefs, B.; Karmel, H. J.; Nyakiti, L. O.; Gaskill, D. K.; Hersam, M. C.; Zegenhagen, J.; Bedzyk, M. J. Chemically Resolved Interface Structure of Epitaxial Graphene on $\mathrm{SiC}(0001)$. Phys. Rev. Lett. 2013, 111, 215501.

(12) Conrad, M.; Rault, J.; Utsumi, Y.; Garreau, Y.; Vlad, A.; Coati, A.; Rueff, J.-P.; Miceli, P. F.; Conrad, E. H. Structure and evolution of semiconducting buffer graphene grown on $\mathrm{SiC}(0001)$. Phys. Rev. B: Condens. Matter Mater. Phys. 2017, 96, 195304.

(13) Dangwal Pandey, A.; Krausert, K.; Franz, D.; Grånäs, E.; Shayduk, R.; Müller, P.; Keller, T. F.; Noei, H.; Vonk, V.; Stierle, A. Single orientation graphene synthesized on iridium thin films grown by molecular beam epitaxy. J. Appl. Phys. 2016, 120, 075304.

(14) Emery, J. D.; Wheeler, V. D.; Johns, J. E.; McBriarty, M. E.; Detlefs, B.; Hersam, M. C.; Gaskill, D. K.; Bedzyk, M. J. Structural consequences of hydrogen intercalation of epitaxial graphene on $\mathrm{SiC}(0001)$. Appl. Phys. Lett. 2014, 105, 161602.

(15) Kowalski, G.; Tokarczyk, M.; Dąbrowski, P.; Ciepielewski, P.; Możdżonek, M.; Strupiński, W.; Baranowski, J. M. New X-ray insight into oxygen intercalation in epitaxial graphene grown on $4 \mathrm{H}$ $\mathrm{SiC}(0001)$. J. Appl. Phys. 2015, 117, 105301.

(16) Drnec, J.; Vlaic, S.; Carlomagno, I.; Gonzalez, C. J.; Isern, H.; Carlà, F.; Fiala, R.; Rougemaille, N.; Coraux, J.; Felici, R. Surface alloying upon Co intercalation between graphene and $\operatorname{Ir}(111)$. Carbon 2015, 94, 554-559.

(17) Campbell, G. P.; Kiraly, B.; Jacobberger, R. M.; Mannix, A. J.; Arnold, M. S.; Hersam, M. C.; Guisinger, N. P.; Bedzyk, M. J. Epitaxial graphene-encapsulated surface reconstruction of $\mathrm{Ge}(110)$. Phys. Rev. Materials 2018, 2, 044004.

(18) Zhou, L.; Fox, L.; Włodek, M.; Islas, L.; Slastanova, A.; Robles, E.; Bikondoa, O.; Harniman, R.; Fox, N.; Cattelan, M.; Briscoe, W. H. Surface structure of few layer graphene. Carbon 2018, 136, 255-261.

(19) Kühne, M.; Paolucci, F.; Popovic, J.; Ostrovsky, P. M.; Maier, J.; Smet, J. H. Ultrafast lithium diffusion in bilayer graphene. Nat. Nanotechnol. 2017, 12, 895-900.

(20) Parratt, L. G. Surface Studies of Solids by Total Reflection of XRays. Phys. Rev. 1954, 95, 359-369.

(21) Novoselov, K. S.; Geim, A. K.; Morozov, S. V.; Jiang, D.; Zhang, Y.; Dubonos, S. V.; Grigorieva, I. V.; Firsov, A. A. Electric Field Effect in Atomically Thin Carbon Films. Science 2004, 306, 666-669.

(22) Dean, C. R.; Young, A. F.; Meric, I.; Lee, C.; Wang, L.; Sorgenfrei, S.; Watanabe, K.; Taniguchi, T.; Kim, P.; Shepard, K. L.; Hone, J. Boron nitride substrates for high-quality graphene electronics. Nat. Nanotechnol. 2010, 5, 722-726.

(23) Bonaccorso, F.; Lombardo, A.; Hasan, T.; Sun, Z.; Colombo, L.; Ferrari, A. C. Production and processing of graphene and $2 \mathrm{~d}$ crystals. Mater. Today 2012, 15, 564-589.

(24) Chason, E.; Mayer, T. M. Thin film and surface characterization by specular X-ray reflectivity. Crit. Rev. Solid State Mater. Sci. 1997, 22, 1-67.

(25) Springer Handbook of Condensed Matter and Materials Data; Martienssen, W., Warlimont, H., Eds.; Springer: Berlin, 2005.

(26) Cullen, W. G.; Yamamoto, M.; Burson, K. M.; Chen, J. H.; Jang, C.; Li, L.; Fuhrer, M. S.; Williams, E. D. High-Fidelity
Conformation of Graphene to $\mathrm{SiO}_{2}$ Topographic Features. Phys. Rev. Lett. 2010, 105, 215504.

(27) Kojima, I.; Li, B.; Fujimoto, T. XRR and XPS studies of $\mathrm{SiO}_{2}$ thin films formed by r.f. magnetron sputtering. Surf. Interface Anal. 1999, 28, 267-270.

(28) Krumrey, M.; Hoffmann, M.; Ulm, G.; Hasche, K.; ThomsenSchmidt, P. Thickness determination for $\mathrm{SiO}_{2}$ films on $\mathrm{Si}$ by X-ray reflectometry at the Si K edge. Thin Solid Films 2004, 459, 241-244.

(29) McCann, E.; Koshino, M. The electronic properties of bilayer graphene. Rep. Prog. Phys. 2013, 76, 056503.

(30) Ishigami, M.; Chen, J. H.; Cullen, W. G.; Fuhrer, M. S.; Williams, E. D. Atomic Structure of Graphene on $\mathrm{SiO}_{2}$. Nano Lett. 2007, 7, 1643-1648.

(31) Nair, J. R.; Gerbaldi, C.; Destro, M.; Bongiovanni, R.; Penazzi, N. Methacrylic-based solid polymer electrolyte membranes for lithium-based batteries by a rapid UV-curing process. React. Funct. Polym. 2011, 71, 409-416.

(32) Kühne, M.; Börrnert, F.; Fecher, S.; Ghorbani-Asl, M.; Biskupek, J.; Samuelis, D.; Krasheninnikov, A. V.; Kaiser, U.; Smet, J. H. Reversible superdense ordering of lithium between two graphene sheets. Nature 2018, 564, 234-239.

(33) Fischer, J. E. In Chemical Physics of Intercalation; Legrand, A. P., Flandrois, S., Eds.; NATO ASI Series. Series B: Physics; Springer Science+Business Media: New York, 1987; Vol. 172, pp 59-78.

(34) Dahn, J. R. Phase diagram of $\mathrm{Li}_{x} \mathrm{C}_{6}$. Phys. Rev. B: Condens. Matter Mater. Phys. 1991, 44, 9170-9177.

(35) Villars, P., Ed.; PAULING FILE. In Inorganic Solid Phases; SpringerMaterials (online database); Springer: Heidelberg; Springer Berlin Heidelberg \& Material Phases Data System (MPDS), Switzerland; National Institute for Materials Science (NIMS), Japan, 2016; p Dataset ID sd_0377652, (accessed Feb 03, 2018).

(36) Enoki, T.; Endo, M.; Suzuki, M. Graphite Intercalation Compounds and Applications; Oxford University Press: New York, 2003.

(37) Heß, M. Kinetics and stage transitions of graphite for lithium-ion batteries. Ph.D. Thesis, ETH Zürich/PSI, Diss. No. 21240, 2013.

(38) Nagashio, K.; Yamashita, T.; Nishimura, T.; Kita, K.; Toriumi, A. Electrical transport properties of graphene on $\mathrm{SiO}_{2}$ with specific surface structures. J. Appl. Phys. 2011, 110, 024513.

(39) Moss, S. C.; Moret, R. In Graphite Intercalation Compounds I; Zabel, H., Solin, S. A., Eds.; Springer Series in Materials Science; Springer: Berlin, 1990; Vol. 14, pp 5-58.

(40) Dahn, J. R.; Fong, R.; Spoon, M. J. Suppression of staging in lithium-intercalated carbon by disorder in the host. Phys. Rev. B: Condens. Matter Mater. Phys. 1990, 42, 6424-6432.

(41) O’Hara, A.; Kahn, R. E.; Zhang, Y.-Y.; Pantelides, S. T. Defectmediated leakage in lithium intercalated bilayer graphene. AIP Adv. 2017, 7, 045205.

(42) Wan, J.; Lacey, S. D.; Dai, J.; Bao, W.; Fuhrer, M. S.; Hu, L. Tuning two-dimensional nanomaterials by intercalation: materials, properties and applications. Chem. Soc. Rev. 2016, 45, 6742-6765.

(43) Zhao, S. Y. F.; Elbaz, G. A.; Bediako, D. K.; Yu, C.; Efetov, D. K.; Guo, Y.; Ravichandran, J.; Min, K.-A.; Hong, S.; Taniguchi, T.; Watanabe, K.; Brus, L. E.; Roy, X.; Kim, P. Controlled Electrochemical Intercalation of Graphene/h-BN van der Waals Heterostructures. Nano Lett. 2018, 18, 460-466.

(44) Bediako, D. K.; Rezaee, M.; Yoo, H.; Larson, D. T.; Zhao, S. Y. F.; Taniguchi, T.; Watanabe, K.; Brower-Thomas, T. L.; Kaxiras, E.; Kim, P. Heterointerface effects in the electrointercalation of van der Waals heterostructures. Nature 2018, 558, 425-429. 